\title{
Half-Sweep Arithmetic Mean Method with High-Order Newton-Cotes Quadrature Schemes to Solve Linear Second Kind Fredholm Equations
}

\author{
Mohana Sundaram Muthuvalu and Jumat Sulaiman* \\ School of Science and Technology, Universiti Malaysia Sabah, Locked Bag 2073, 88999 Kota Kinabalu, Sabah, Malaysia. \\ *To whom correspondence should be addressed. E-mail: jumat@ums.edu.my
}

Received: 11 November 2008, Revised: 7 January 2009

Online Publication: 26 May 2009

http://dx.doi.org/10.11113/mifas.v5n1.282

\section{ABSTRACT}

The main purpose of this paper is to examine the effectiveness of the Half-Sweep Arithmetic Mean (HSAM) method in solving the dense linear systems generated from the discretization of the linear Fredholm integral equations of the second kind. In addition, the applications of the various orders of closed Newton-Cotes quadrature discretization schemes will be investigate in order to form linear systems. Furthermore, the basic formulation and implementation for the proposed method are also presented. Some illustrative examples are given to point out the efficiency of the proposed method.

| Linear Fredholm equations | Quadrature scheme | Half-Sweep Arithmetic Mean method |

\section{Introduction}

Two-stage iterative methods are one of the widely used and successful classes of numerical algorithms to solve any system of linear algebraic equations that is generated by using approximation equations. Actually, there are many two-stage iterative methods can be considered such as the Alternating Group Explicit (AGE) [1], Iterative Alternating Decomposition Explicit (IADE) [2], Reduced Iterative Alternating Decomposition Explicit (RIADE) [3], Half-Sweep Iterative Alternating Decomposition Explicit (HSIADE) [4], Quarter-Sweep Iterative Alternating Decomposition Explicit (QSIADE) [5], Block Jacobi [6] and Arithmetic Mean (AM) [7] methods.

In 2004, Sulaiman et al. [8] modified the standard AM method also named as the Full-Sweep Arithmetic Mean (FSAM) method by combining the concept of the half-sweep iteration method and then called as the HalfSweep Arithmetic Mean (HSAM) method. The concept of the half-sweep iteration method is introduced via the Explicit Decoupled Group (EDG) iterative method for solving two-dimensional Poisson equations [9]. Further studies of the HSAM method have been also conducted to solve Poisson's equation using finite element [10] and fourth-order finite difference [11] methods. Besides that, HSAM method also have been applied to solve linear systems generated from the discretization of the Fredholm integral equations, see [12-14]. In this paper, the 
application of the FSAM and HSAM methods using the approximation equation based on quadrature methods for solving linear second kind Fredholm integral equations is examined. follows

Generally, linear second kind integral equations of Fredholm type in the standard form can be defined as

$$
\lambda y(x)-\int_{\Gamma} K(x, t) y(t) d t=f(x), \Gamma=[a, b] \quad \lambda \neq 0
$$

where the parameter $\lambda$, kernel $K(x, t) \in L_{2}[a, b] \times[a, b]$ and free term $f(x) \in L_{2}[a, b]$ are given, and $y(x)$ is the unknown function to be determined. The kernel function $K(x, t)$ is assumed to be absolutely integrable and satisfy other properties that are sufficient to imply the Fredholm alternative theorem.

\section{Theorem (Fredholm alternative) [15]}

Let $\chi$ be a Banach space and let $\kappa: \chi \rightarrow \chi$ be compact. Then the equation $(\lambda-\kappa) y=f, \lambda \neq 0$ has a unique solution $x \in \chi$ if and only if the homogeneous equation $(\lambda-\kappa) z=0$ has only the trivial solution $z=0$. In such a case, the operator $\lambda-\kappa: \chi \underset{\text { onto }}{\rightarrow} \chi$ has a bounded inverse $(\lambda-\kappa)^{-1}$.

\section{Definition (Compact operators) [15]}

Let $\chi$ and $\mathrm{Y}$ be normed vector space and let $\kappa: \chi \rightarrow \mathrm{Y}$ be linear. Then $\kappa$ is compact if the set

$$
\{k x \mid\|x\| x \leq 1\}
$$

has compact closure in $\mathrm{Y}$. This is equivalent to saying that for every bounded sequence $\left\{x_{n}\right\} \subset \chi$, the sequence $\left\{\kappa x_{n}\right\}$ has a subsequence that is convergent to some point in $\mathrm{Y}$. Compact operators are also called completely continuous operators.

The remainder of this paper is organized in following way. In next section, the formulation of the full- and half-sweep quadrature approximation equations based on repeated Newton-Cotes schemes is described. The latter section of this paper will discuss the formulations of the FSAM and HSAM methods and some numerical results will be shown to indicate the effectiveness of the HSAM method. Meanwhile, conclusion and directions of the future works are drawn in final section.

\section{Half-Sweep Quadrature Approximation Equations}

In many application areas, numerical approaches were used widely to solve Fredholm integral equations of the second kind than the analytical methods. For the solution of a linear integral equation, reduction of integral equation to the solution of system of linear algebraic equations is the basic concept used to solve the problems. There are many methods can be used to discretize the linear Fredholm integral equations into linear systems such as quadrature [15-19] and projection [20-23] methods.

In this paper, however, discretization schemes based on quadrature method were used to discretize the linear Fredholm integral equations of the second kind in order to form a linear system. Quadrature formulas in general have the form

$$
\int_{a}^{b} y(t) d t=\sum_{j=0}^{n} A_{j} y\left(t_{j}\right)+\varepsilon_{n}(y)
$$


where $t_{j}, j=0,1, \ldots, n$ are the abscissas of the partition points of the integration interval $[a, b]$ or quadrature (interpolation) nodes, $A_{j}, j=0,1, \ldots, n$ are numerical coefficients that do not depend on the function $y(t)$ and $\varepsilon_{n}(y)$ is the truncation error of Eq. (2).

To facilitate in formulating the full- and half-sweep approximation equations for linear Fredholm equation of the second kind, further discussion will be restricted onto Newton-Cotes quadrature method, which is based on interpolation formulas with equally spaced data. In this paper, four different schemes in Newton-Cotes method such as repeated trapezoidal (RT), repeated Simpson's $\frac{1}{3}$ (RS1), repeated Simpson's $\frac{3}{8}$ (RS2) and repeated Boole (RB) will be applied to discretize the problem. RT, RS1, RS2 and RB are first, second, third and fourth order schemes respectively. Meanwhile, Figure 1 shows the finite grid networks in order to form the full- and half-sweep approximation methods for problem (1).

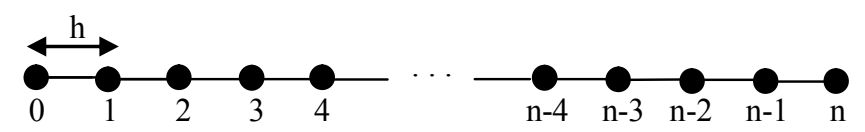

a)

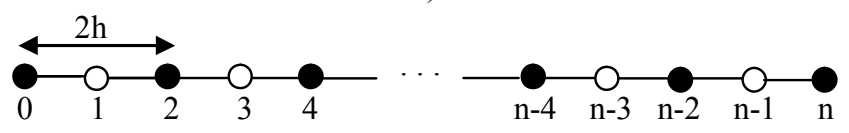

b)

Figure 1: a) and b) show distribution of uniform node points for the full- and half-sweep cases respectively.

Based on the Figure 1, the full- and half-sweep iterative methods will compute approximate values onto node points of type only until the convergence criterion is reached. Then other approximate solutions at remaining points (points of the different type, $\bigcirc$ ) are obtained using the direct method [9,24,25]. By applying Eq. (2) into Eq. (1) and neglecting the error, $\varepsilon_{n}(y)$ a system of linear algebraic equations can be formed for approximation values $y(x)$ at the nodes $x_{0}, x_{1}, \ldots, x_{n}$. The following linear system generated using the Newton-Cotes quadrature method can be easily shown as

where

$$
M \boldsymbol{y}=\boldsymbol{f}
$$

$$
\begin{aligned}
& M=\left[\begin{array}{ccccc}
\lambda-A_{0} K_{0,0} & -A_{p} K_{0, p} & -A_{2 p} K_{0,2 p} & \cdots & -A_{n} K_{0, n} \\
-A_{0} K_{p, 0} & \lambda-A_{p} K_{p, p} & -A_{2 p} K_{p, 2 p} & \cdots & -A_{n} K_{p, n} \\
-A_{0} K_{2 p, 0} & -A_{p} K_{2 p, p} & \lambda-A_{2 p} K_{2 p, 2 p} & \cdots & -A_{n} K_{2 p, n} \\
\vdots & \vdots & \vdots & \ddots & \vdots \\
-A_{0} K_{n, 0} & -A_{p} K_{n, p} & -A_{2 p} K_{n, 2 p} & \cdots & \lambda-A_{n} K_{n, n}
\end{array}\right]\left(\left(\frac{n}{p}\right)+1\right) \times\left(\left(\frac{n}{p}\right)+1\right) \\
& \boldsymbol{y}=\left[\begin{array}{lllllll}
y_{0} & y_{p} & y_{2 p} & \cdots & y_{n-2 p} & y_{n-p} & y_{n}
\end{array}\right]^{T}
\end{aligned}
$$

and

$$
\boldsymbol{f}=\left[\begin{array}{lllllll}
f_{0} & f_{p} & f_{2 p} & \cdots & f_{n-2 p} & f_{n-p} & f_{n}
\end{array}\right]^{T} .
$$


Based on repeated trapezoidal, repeated Simpson's $\frac{1}{3}$, repeated Simpson's $\frac{3}{8}$ and repeated Boole schemes, numerical coefficient $A_{j}$ satisfied following relations respectively.

$$
\begin{gathered}
A_{j}=\left\{\begin{array}{l}
\frac{1}{2} p h, \quad j=0, n \\
p h, \quad \text { otherwise }
\end{array}\right. \\
A_{j}= \begin{cases}\frac{1}{3} p h, \quad j=0, n \\
\frac{4}{3} p h, \quad j=p, 3 p, 5 p, \cdots, n-p \\
\frac{2}{3} p h, \quad \text { otherwise }\end{cases} \\
A_{j}= \begin{cases}\frac{3}{8} p h, \quad j=0, n \\
\frac{3}{4} p h, \quad j=3 p, 6 p, 9 p, \cdots, n-3 p \\
\frac{9}{8} p h, \quad \text { otherwise }\end{cases} \\
A_{j}= \begin{cases}\frac{14}{45} p h, \quad j=0, n \\
\frac{24}{45} p h, \quad j=2 p, 6 p, 10 p, \cdots, n-2 p \\
\frac{28}{45} p h, \quad j=4 p, 8 p, 12 p, \cdots, n-4 p \\
\frac{64}{45} p h, \quad \text { otherwise }\end{cases}
\end{gathered}
$$

where the constant step size, $h$ is defined as

$$
h=\frac{b-a}{n} .
$$

$n$ is the number of subintervals in the interval $[a, b]$ and then consider the discrete set of points be given as $x_{i}=a+i h$. The value of $p$, which corresponds to 1 and 2 , represents the full- and half-sweep cases respectively.

\section{Derivation of the Half-Sweep Arithmetic Mean Method}

As afore-mentioned in the previous section, the HSAM method is one of two-stage iterative methods. It means that the iterative process for this method consists of solving two independent systems such as $\tilde{\boldsymbol{y}}$ and $\hat{\boldsymbol{y}}$. To develop formulation of HSAM and FSAM methods, matrix $M$ needs to be decomposed into

$$
M=L+D+T
$$

where $L, D$ and $T$ are strictly lower triangular, diagonal and strictly upper triangular matrices respectively. Thus, for real positive acceleration parameter, $r$, the general scheme for both AM methods is given by 


$$
\begin{aligned}
& (D+r L) \tilde{\boldsymbol{y}}=((1-r) D-r T) \boldsymbol{y}^{(k)}+r \boldsymbol{f} \\
& (D+r T) \hat{\boldsymbol{y}}=((1-r) D-r L) \boldsymbol{y}^{(k)}+r \boldsymbol{f} \\
& \boldsymbol{y}^{(k+1)}=\frac{1}{2}(\tilde{\boldsymbol{y}}+\hat{\boldsymbol{y}})
\end{aligned}
$$

In Eq. (10), $\boldsymbol{y}^{(k)}$ represents as an unknown vector at the $k^{\text {th }}$ iteration and $\boldsymbol{y}^{(0)}$ is an initial vector approximation to the solution $y$ of (9). Practically, the value of $r$ will be determined by implementing some computer programs and then choose one value of $r$, where its number of iterations is the smallest. By determining values of matrices $L, D$ and $T$ as stated in Eq. (9), the general algorithm for FSAM and HSAM schemes in Eq. (10) would be described in Algorithm 1. The FSAM and HSAM algorithms are explicitly performed by using all equations at level (1) and (2) alternatively until the specified convergence criterion is satisfied.

Algorithm 1: FSAM and HSAM schemes

i) Level (1)

For $\quad i=0, p, 2 p, \cdots, n-2 p, n-p, n$

Calculate

$$
\tilde{y}_{i} \leftarrow \begin{cases}\left((1-r)\left(\lambda-A_{i} K_{i i}\right) y_{i}{ }^{(k)}+r \sum_{j=p}^{n} A_{j} K_{i j} y_{j}{ }^{(k)}+r f_{i}\right) /\left(\lambda-A_{i} K_{i i}\right) & , i=0 \\ \left((1-r)\left(\lambda-A_{i} K_{i i}\right) y_{i}{ }^{(k)}+r \sum_{j=0}^{n-p} A_{j} K_{i j} \tilde{y}_{j}+r f_{i}\right) /\left(\lambda-A_{i} K_{i i}\right) & , i=n \\ \left((1-r)\left(\lambda-A_{i} K_{i i}\right) y_{i}{ }^{(k)}+r \sum_{j=0}^{i-p} A_{j} K_{i j} \tilde{y}_{j}+r \sum_{j=i+p}^{n} A_{j} K_{i j} y_{j}{ }^{(k)}+r f_{i}\right) /\left(\lambda-A_{i} K_{i i}\right) & , \text { others }\end{cases}
$$

ii) Level (2)

For $i=n, n-p, n-2 p \cdots, 2 p, p, 0$

Calculate

$$
\hat{y}_{i} \leftarrow \begin{cases}\left((1-r)\left(\lambda-A_{i} K_{i i}\right) y_{i}{ }^{(k)}+r \sum_{j=1}^{n} A_{j} K_{i j} \hat{y}_{j}+r f_{i}\right) /\left(\lambda-A_{i} K_{i i}\right) & , i=0 \\ \left((1-r)\left(\lambda-A_{i} K_{i i}\right) y_{i}{ }^{(k)}+r \sum_{j=0}^{n-p} A_{j} K_{i j} y_{j}{ }^{(k)}+r f_{i}\right) /\left(\lambda-A_{i} K_{i i}\right) & , i=n \\ \left((1-r)\left(\lambda-A_{i} K_{i i}\right) y_{i}{ }^{(k)}+r \sum_{j=0}^{i-p} A_{j} K_{i j} y_{j}{ }^{(k)}+r \sum_{j=i+p}^{n} A_{j} K_{i j} \hat{y}_{j}+r f_{i}\right) /\left(\lambda-A_{i} K_{i i}\right) & , \text { others }\end{cases}
$$


iii) For $i=0, p, 2 p, \cdots, n-2 p, n-p, n$

Calculate

$$
y_{i}^{(k+1)} \leftarrow \frac{1}{2}\left(\tilde{y}_{i}+\hat{y}_{i}\right)
$$

\section{Numerical Simulations}

In this section, some numerical simulations have been conducted to assess the effectiveness of the proposed method through three parameters such as number of iterations, execution time and maximum absolute error. In comparison, the Full-Sweep Gauss-Seidel (FSGS) method act as the control of comparison of numerical results. In the implementation of the iterative methods, the convergence test considered the tolerance error, $\varepsilon$ of $10^{-10}$. As mentioned above, Newton-cotes quadrature schemes are used to discretize and to form a linear system for the following example.

\section{Example 1 (Wang [26])}

Consider the integral equation

$$
y(x)-\int_{0}^{1}\left(4 x t-x^{2}\right) y(t) d t=x
$$

and the exact solution of problem (11) is given by

$$
y(x)=24 x-9 x^{2}
$$

Results of numerical experiments, which were obtained from implementations of the iterative methods for Example 1, have been recorded in Table 1.

Example 2 (Polyanin \& Manzhirov [19])

Consider the integral equation

$$
y(x)=x^{6}-5 x^{3}+x+10+\int_{0}^{1}\left(x^{2}+t^{2}\right) y(t) \cdot d t
$$

Exact solution of the problem is

$$
y(x)=x^{6}-5 x^{3}+\frac{1045}{28} x^{2}+x+\frac{2141}{84} .
$$

For Example 2, numerical results of FSGS, HSGS, FSAM and HSAM methods have been recorded in Table 2.

\section{Conclusions}

In the previous section, it has shown that the quadrature approximation equations based on Newton-Cotes formulas can be easily formulated and rewritten in general form as shown in Eq. (3). Through numerical results obtained in Tables 1 and 2, clearly it shows that by applying the AM methods can reduce the number of iterations compared to Gauss-Seidel (GS) methods. Through the numerical results obtained for Example 1 by using RT and RS2 schemes, number of iterations for FSAM and HSAM methods have declined approximately 56.48 - 56.77\% and 56.70 - 56.99\% respectively compared to FSGS method. Number of iterations for FSAM and HSAM methods with RS1 and RB schemes for Example 1 have decreased by $56.48-56.70 \%$ and $56.54-56.99 \%$ respectively compared to FSGS method. Meanwhile, number of iterations for both AM iterative methods for 
Table 1: Comparison of a number of iterations, execution time (seconds) and maximum absolute error for the iterative methods using RT, RS1, RS2 and RB discretization schemes (Example 1).

\begin{tabular}{|c|c|c|c|c|c|c|c|c|c|c|c|c|c|}
\hline \multirow{2}{*}{$\begin{array}{c}\text { Mesh } \\
\text { size }\end{array}$} & \multirow{2}{*}{ Methods } & \multicolumn{4}{|c|}{ Number of iterations } & \multicolumn{4}{|c|}{ Execution time (seconds) } & \multicolumn{4}{|c|}{ Maximum absolute error } \\
\hline & & FSGS & HSGS & FSAM & HSAM & FSGS & HSGS & FSAM & HSAM & FSGS & HSGS & FSAM & HSAM \\
\hline \multirow{4}{*}{120} & RT & 192 & 189 & 83 & 83 & 0.66 & 0.16 & 0.60 & 0.11 & 8.5466 E-3 & $3.4245 \mathrm{E}-2$ & 8.5466 E-3 & $3.4245 \mathrm{E}-2$ \\
\hline & RS1 & 191 & 188 & 83 & 83 & 0.71 & 0.16 & 0.60 & 0.11 & $7.4883 \mathrm{E}-10$ & $6.2500 \mathrm{E}-4$ & $2.3642 \mathrm{E}-10$ & $6.2500 \mathrm{E}-4$ \\
\hline & RS2 & 192 & 189 & 83 & 83 & 0.77 & 0.17 & 0.61 & 0.16 & $6.8299 \mathrm{E}-10$ & $6.2500 \mathrm{E}-4$ & 2.3796 E-10 & $6.2500 \mathrm{E}-4$ \\
\hline & RB & 191 & 188 & 83 & 83 & 0.77 & 0.22 & 0.66 & 0.16 & $7.2890 \mathrm{E}-10$ & $6.2500 \mathrm{E}-4$ & 2.3473 E-10 & $6.2500 \mathrm{E}-4$ \\
\hline \multirow{4}{*}{240} & RT & 193 & 192 & 84 & 83 & 2.74 & 0.66 & 2.47 & 0.60 & $2.1357 \mathrm{E}-3$ & $8.5466 \mathrm{E}-3$ & $2.1357 \mathrm{E}-3$ & 8.5466 E-3 \\
\hline & RS1 & 193 & 191 & 84 & 83 & 2.91 & 0.71 & 2.58 & 0.61 & 7.2615 E-10 & $1.5625 \mathrm{E}-4$ & 1.4414 E-10 & $1.5625 \mathrm{E}-4$ \\
\hline & RS2 & 193 & 192 & 84 & 83 & 2.91 & 0.77 & 2.58 & 0.65 & 7.3756 E-10 & $1.5625 \mathrm{E}-4$ & $1.4114 \mathrm{E}-10$ & $1.5625 \mathrm{E}-4$ \\
\hline & RB & 193 & 191 & 84 & 83 & 3.19 & 0.82 & 2.74 & 0.71 & 7.1661 E-10 & $1.5625 \mathrm{E}-4$ & 1.4486 E-10 & $1.5625 \mathrm{E}-4$ \\
\hline \multirow{4}{*}{480} & RT & 194 & 193 & 84 & 84 & 10.98 & 2.74 & 9.89 & 2.36 & 5.3387 E-4 & $2.1357 \mathrm{E}-3$ & $5.3387 \mathrm{E}-4$ & $2.1357 \mathrm{E}-3$ \\
\hline & RS1 & 194 & 193 & 84 & 84 & 11.70 & 2.91 & 10.16 & 2.58 & 7.1563 E-10 & 3.9063 E-5 & $1.4801 \mathrm{E}-10$ & 3.9063 E-5 \\
\hline & RS2 & 194 & 193 & 84 & 84 & 11.75 & 2.92 & 10.22 & 2.59 & 7.2118 E-10 & 3.9063 E-5 & 1.4657 E-10 & 3.9063 E-5 \\
\hline & RB & 194 & 193 & 84 & 84 & 12.69 & 3.41 & 10.99 & 2.91 & 7.1093 E-10 & $3.9063 \mathrm{E}-5$ & 1.4836 E-10 & $3.9063 \mathrm{E}-5$ \\
\hline \multirow{4}{*}{960} & RT & 194 & 194 & 84 & 84 & 43.83 & 11.26 & 39.60 & 9.50 & 1.3346 E-4 & $5.3387 \mathrm{E}-4$ & 1.3346 E-4 & $5.3387 \mathrm{E}-4$ \\
\hline & RS1 & 194 & 194 & 84 & 84 & 46.64 & 11.76 & 40.43 & 10.16 & 7.5521 E-10 & 9.7663 E-6 & 1.5014 E-10 & 9.7658 E-6 \\
\hline & RS2 & 194 & 194 & 84 & 84 & 47.02 & 11.76 & 40.65 & 10.22 & 7.5812 E-10 & 9.7663 E-6 & $1.4953 \mathrm{E}-10$ & 9.7658 E-6 \\
\hline & RB & 194 & 194 & 84 & 84 & 50.70 & 13.63 & 43.89 & 11.53 & 7.5274 E-10 & 9.7663 E-6 & $1.5034 \mathrm{E}-10$ & 9.7658 E-6 \\
\hline \multirow{4}{*}{1200} & RT & 194 & 194 & 84 & 84 & 68.49 & 17.58 & 62.01 & 14.89 & 8.5416 E-5 & $3.4167 \mathrm{E}-4$ & 8.5417 E-5 & $3.4167 \mathrm{E}-4$ \\
\hline & RS1 & 194 & 194 & 84 & 84 & 72.94 & 18.29 & 63.22 & 15.93 & 7.6332 E-10 & 6.2507 E-6 & 1.5052 E-10 & $6.2501 \mathrm{E}-6$ \\
\hline & RS2 & 194 & 194 & 84 & 84 & 73.55 & 18.34 & 63.55 & 15.98 & 7.6567 E-10 & 6.2507 E-6 & 1.5005 E-10 & $6.2501 \mathrm{E}-6$ \\
\hline & RB & 194 & 194 & 84 & 84 & 79.04 & 21.25 & 68.54 & 18.07 & 7.6132 E-10 & $6.2507 \mathrm{E}-6$ & 1.5068 E-10 & $6.2501 \mathrm{E}-6$ \\
\hline
\end{tabular}


Table 2: Comparison of a number of iterations, execution time (seconds) and maximum absolute error for the iterative methods using RT, RS1, RS2 and RB discretization schemes (Example 2).

\begin{tabular}{|c|c|c|c|c|c|c|c|c|c|c|c|c|c|}
\hline \multirow{2}{*}{$\begin{array}{l}\text { Mesh } \\
\text { size }\end{array}$} & \multirow{2}{*}{ Methods } & \multicolumn{4}{|c|}{ Number of iterations } & \multicolumn{4}{|c|}{ Execution time (seconds) } & \multicolumn{4}{|c|}{ Maximum absolute error } \\
\hline & & FSGS & HSGS & FSAM & HSAM & FSGS & HSGS & FSAM & HSAM & FSGS & HSGS & FSAM & HSAM \\
\hline \multirow{4}{*}{120} & RT & 55 & 55 & 32 & 32 & 0.18 & 0.05 & 0.17 & 0.04 & $8.6975 \mathrm{E}-3$ & $3.7011 \mathrm{E}-2$ & $8.6975 \mathrm{E}-3$ & $3.7011 \mathrm{E}-2$ \\
\hline & RS1 & 55 & 55 & 32 & 32 & 0.19 & 0.05 & 0.18 & 0.04 & $1.8143 \mathrm{E}-7$ & $2.5842 \mathrm{E}-3$ & $1.8150 \mathrm{E}-7$ & $2.5842 \mathrm{E}-3$ \\
\hline & RS2 & 55 & 55 & 32 & 32 & 0.19 & 0.06 & 0.18 & 0.04 & $4.0839 \mathrm{E}-7$ & $2.5856 \mathrm{E}-3$ & $4.0845 \mathrm{E}-7$ & $2.5856 \mathrm{E}-3$ \\
\hline & RB & 55 & 55 & 32 & 32 & 0.22 & 0.07 & 0.20 & 0.05 & $1.1615 \mathrm{E}-10$ & 2.5831 E-3 & $6.8426 \mathrm{E}-11$ & $2.5831 \mathrm{E}-3$ \\
\hline \multirow{4}{*}{240} & RT & 56 & 55 & 32 & 32 & 1.25 & 0.20 & 1.23 & 0.18 & $2.1741 \mathrm{E}-3$ & $9.2970 \mathrm{E}-3$ & 2.1741 E-3 & $9.2970 \mathrm{E}-3$ \\
\hline & RS1 & 56 & 55 & 32 & 32 & 1.27 & 0.21 & 1.24 & 0.18 & $1.1238 \mathrm{E}-8$ & 6.4693 E-4 & $1.1251 \mathrm{E}-8$ & 6.4693 E-4 \\
\hline & RS2 & 56 & 55 & 32 & 32 & 1.28 & 0.21 & 1.24 & 0.19 & $2.5423 \mathrm{E}-8$ & 6.4702 E-4 & 2.5437 E-8 & 6.4702 E-4 \\
\hline & RB & 56 & 55 & 32 & 32 & 1.35 & 0.30 & 1.30 & 0.23 & $1.0991 \mathrm{E}-10$ & 6.4686 E-4 & $1.0415 \mathrm{E}-10$ & 6.4686 E-4 \\
\hline \multirow{4}{*}{480} & RT & 56 & 56 & 32 & 32 & 3.56 & 1.28 & 3.48 & 1.25 & $5.4352 \mathrm{E}-4$ & $2.3300 \mathrm{E}-3$ & 5.4352 E-4 & $2.3300 \mathrm{E}-3$ \\
\hline & RS1 & 56 & 56 & 32 & 32 & 3.66 & 1.30 & 3.51 & 1.26 & 5.8823 E-10 & $1.6185 \mathrm{E}-4$ & $6.1321 \mathrm{E}-10$ & $1.6185 \mathrm{E}-4$ \\
\hline & RS2 & 56 & 56 & 32 & 32 & 3.69 & 1.33 & 3.54 & 1.28 & 1.4742 E-9 & $1.6186 \mathrm{E}-4$ & 1.4930 E-9 & $1.6186 \mathrm{E}-4$ \\
\hline & RB & 56 & 56 & 32 & 32 & 3.80 & 1.50 & 3.74 & 1.41 & $1.2051 \mathrm{E}-10$ & 1.6185 E-4 & $1.0556 \mathrm{E}-10$ & $1.6185 \mathrm{E}-4$ \\
\hline \multirow{4}{*}{960} & RT & 56 & 56 & 32 & 32 & 14.43 & 3.80 & 14.10 & 3.57 & 1.3588 E-4 & $5.8325 \mathrm{E}-4$ & $1.3588 \mathrm{E}-4$ & $5.8325 \mathrm{E}-4$ \\
\hline & RS1 & 56 & 56 & 32 & 32 & 14.50 & 3.90 & 14.16 & 3.59 & 8.3052 E-11 & 4.0479 E-5 & 6.1889 E-11 & 4.0480 E-5 \\
\hline & RS2 & 56 & 56 & 32 & 32 & 14.59 & 3.95 & 14.20 & 3.60 & 3.8873 E-11 & 4.0480 E-5 & 3.6176 E-11 & 4.0480 E-5 \\
\hline & RB & 56 & 56 & 32 & 32 & 14.70 & 4.17 & 14.44 & 3.75 & $1.2582 \mathrm{E}-10$ & 4.0479 E-5 & $1.0613 \mathrm{E}-10$ & 4.0479 E-5 \\
\hline \multirow{4}{*}{1200} & RT & 56 & 56 & 32 & 32 & 18.45 & 6.05 & 17.73 & 5.87 & $8.6962 \mathrm{E}-5$ & 3.7338 E-4 & 8.6962 E-5 & $3.7338 \mathrm{E}-4$ \\
\hline & RS1 & 56 & 56 & 32 & 32 & 18.49 & 6.08 & 17.77 & 5.92 & $1.0903 \mathrm{E}-10$ & 2.5909 E-5 & $8.8048 \mathrm{E}-11$ & 2.5909 E-5 \\
\hline & RS2 & 56 & 56 & 32 & 32 & 18.53 & 6.10 & 17.80 & 5.95 & 8.7493 E-11 & 2.5909 E-5 & $6.5183 \mathrm{E}-11$ & 2.5909 E-5 \\
\hline & RB & 56 & 56 & 32 & 32 & 20.12 & 6.39 & 19.69 & 6.13 & $1.2686 \mathrm{E}-10$ & 2.5909 E-5 & $1.0631 \mathrm{E}-10$ & 2.5909 E-5 \\
\hline
\end{tabular}


Example 2 using RT, RS1, RS2 and RB discretization schemes as shown in Table 2 decreased approximately 41.82 - 42.86\% compared with FSGS method. For HSGS method with RT, RS1, RS2 and RB schemes, number of iterations is nearly same to the FSGS method for both examples, refer Tables 1 and 2.

Through the observation in Tables 1 and 2, FSAM and HSAM iterative methods reduce the execution time compared to the FSGS method respectively. Overall, the computational time for HSGS and HSAM methods together with RT, RS1, RS2 and RB is superior compared to the corresponding full-sweep iterative methods. This is due to the computational complexity of the half-sweep iterative methods are approximately $50 \%$ less than fullsweep iterative methods respectively.

In terms of accuracy of numerical solutions obtained, repeated Simpson's $\frac{1}{3}$, repeated Simpson's $\frac{3}{8}$ and repeated Boole schemes are more accurate than the repeated trapezoidal scheme. From the observation of the results obtained, it shows that application of the half-sweep iteration concept reduce the accuracy of the solution for RS1, RS2 and RB discretization schemes compared to the full-sweep cases respectively. Decrement of the accuracy for half-sweep iterative methods is due to the computational technique for calculating the remaining points using direct method as proposed by Abdullah [9].

For future works, this study will be extended to investigate the applications of half-sweep concept with different approach for calculating the remaining points. The mesh sizes which have been proposed in this paper will generate a completed group for each discretization schemes. However, this study will also be proceeded to examine for the case of ungroup.

\section{References}

[1] D.J. Evans and M.S. Sahimi, Ann. Rev. Num. Fluid Mech. and Heat Trans., 2 (1988) 283-389.

[2] M.S. Sahimi, A. Ahmad and A.A. Bakar, International Journal of Computer Mathematics, 47 (1993) $219-$ 229.

[3] M.S. Sahimi and M. Khatim, Pertanika J. Sci. and Technol., 9(1) (2001) 13-20.

[4] J. Sulaiman, M.K. Hasan and M. Othman, Computational and Information Science 2004. Lecture Note on Computer Science, LNCS 3314 (2004) 57-63.

[5] J. Sulaiman, M. Othman and M.K. Hasan, International Journal of Computer Mathematics, 81(12) (2004) 1559-1565.

[6] T. Allahviranloo, E. Ahmady, N. Ahmady and K.S. Alketaby, Applied Mathematics and Computation, 175 (2006) 1217-1228.

[7] V. Ruggiero and E. Galligani, Computer Math. Applic., 20 (1) (1990) 25-28.

[8] J. Sulaiman, M. Othman and M.K. Hasan, Proceedings of the International Conference on Statistics and Mathematics and Its Application in the Development of Science and Technology, (2004) 169-173.

[9] A.R. Abdullah, International Journal Computer Mathematics, 38 (1991) 61-70.

[10] J. Sulaiman, M. Othman and M.K. Hasan, Conference on Applied Mathematics, (2005).

[11] J. Sulaiman, M. Othman and M.K. Hasan, Proceedings of the First IMT-GT Regional Conference on Mathematics, Statistics and Their Applications, (2005) 139-146.

[12] M.S. Muthuvalu and J. Sulaiman, Prosiding Simposium Kebangsaan Sains Matematik ke-15, (2007) 211218.

[13] M.S. Muthuvalu and J. Sulaiman, Proceedings of the First Regional Conference on Computational Science and Technology, (2007) 486-490.

[14] M.S. Muthuvalu and J. Sulaiman, Prosiding Seminar Kebangsaan Matematik dan Masyarakat, (2008) 177183. 
[15] K.E. Atkinson, The Numerical Solution of Integral Equations of the Second Kind, Cambridge University Press, United Kingdom, 1997.

[16] M.A. Abdou, Applied Mathematics and Computation, 137 (2003) 231-243.

[17] C.T.H. Baker, The Numerical Treatment of Integral Equations, Clarendon Press, Oxford, 1977.

[18] D.P. Laurie, Journal of Computational and Applied Mathematics, 127 (2001) 201-217.

[19] A.D. Polyanin and A.V. Manzhirov, Handbook of Integral Equations, CRC Press LLC, Florida, 1998.

[20] K. Maleknejad, R. Mollapourasl and K. Nouri, Applied Mathematics and Computation, 181 (2006) 10001007.

[21] K. Maleknejad and M.T. Kajani, Applied Mathematics and Computation, 145 (2003) 623-629.

[22] A. Golbabai and S. Seifollahi, Applied Mathematics and Computation, 181 (2006) 903-907.

[23] M.H. Reihani and Z. Abadi, Journal of Computational and Applied Mathematics, 200 (2007) 12-20.

[24] A. Ibrahim and A.R. Abdullah, International Journal Computer Mathematics, 58 (1995) 253-256.

[25] J. Sulaiman and A.R. Abdullah, Sains Malaysiana, 28 (1999) 161-172.

[26] W. Wang, Applied Mathematics and Computation, 172 (2006) 946-962. 